\title{
A statistical and distributed packet filter against DDoS attacks in Cloud environment
}

\author{
VIKASH C PANDEY $^{1, *}$, SATEESH K PEDDOJU ${ }^{1}$ and PRACHI S DESHPANDE ${ }^{2}$ \\ ${ }^{1}$ Department of Computer Science and Engineering, Indian Institute of Technology Roorkee, Roorkee 247667, \\ India \\ ${ }^{2}$ Department of Applied Science and Engineering, Indian Institute of Technology Roorkee, Roorkee 247667, \\ India \\ e-mail: v.c.pandey89@gmail.com; sateesh@ieee.org; deprachi3@gmail.com
}

MS received 27 May 2014; revised 12 November 2017; accepted 21 November 2017; published online 14 March 2018

\begin{abstract}
Distributed Denial of Service (DDoS) attacks are a serious threat to Cloud. These attacks consume large amount of resources and increase the service usage cost by a significant factor. Due to multi-tenancy and self-provisioning properties of Cloud, traditional DDoS detection techniques cannot be directly applied. Hence, there is a need for Cloud-specific DDoS detection framework. In this paper, a statistical and distributed network packet filtering model is proposed against DDoS attacks in Cloud. The key idea of this scheme is to distribute multiple packet filters among individual virtual machines, which generate and share collective profile of normal behaviour with a coordinator node at constant intervals. Statistics of selected network attributes construct the normal behaviour profile. Based on the deviation from normal behaviour a decision is made whether to accept or reject the incoming packet. The coordinator node monitors filter and distribute the averaged profile to newly provisioned nodes. Individual profiles have low memory and storage requirements and are updated dynamically. Simulation study indicates the effectiveness of this scheme in detecting DDoS attacks in Cloud.
\end{abstract}

Keywords. Cloud security; network security; anomaly detection; network traffic analysis; DDoS attack detection.

\section{Introduction}

Distributed Denial of Service (DDoS) attack is a type of intrusion that exhausts resources and services of an individual or organisation by sending useless traffic, so that legitimate users are not able to avail the services. DDoS attackers mask their identity by compromising many victim machines and use them to mimic the legitimate network traffic, making it very difficult to identify them. Attacks in 2000 against Yahoo, eBay and CNN [1] proved the disruptiveness of DDoS. Cloud has distributed and open structure and its users have lack of control of their resources, which makes it an extremely attractive target for attackers.

Cloud's multi-tenancy, self-provisioning and pay-asyou-use features can be used by attackers against itself. Use of Amazon's elastic Cloud services in 2011 to attack Sony's online entertainment services [2] is an example, which caused 3 weeks outage of the service. Packet filters can be very valuable to detect the intrusion in an early stage and prevent more harmful activity across the system.

*For correspondence
This paper proposes a framework for detection of DDoS attacks on Cloud. This approach defines a DDoS packet filter that acts as a detection tool using certain characteristic features or attributes of network traffic to filter illegitimate traffic from legitimate ones. Terms features and attributes will be used interchangeably throughout the paper. These filters are positioned across multiple tenants in Cloud. DDoS detectors are placed on each host and corresponding packet filters are distributed across individual virtual machines (VMs). Detectors generate a collective network profile of non-intrusive traffic based on the statistics of selected network attributes. They calculate dependency rating of packets in intrusive period to detect deviation from network profile. Based on this, a decision is made whether to accept or reject the packet. Distributed nature of filter, with automatic provisioning of Cloud, ensures scalability of this model.

The rest of this paper is organized as follows. Section 2 describes the related work. Section 3 explains the framework of proposed packet filtering scheme in Cloud. Performance evaluation of the proposed scheme is presented in section 4 . The paper is concluded in section 5 . 


\section{Related work}

DDoS attacks have been classified on various parameters [3] by Mirkovic J and Reiher P. For defense against DDoS attacks, preventive or reactive strategies can be used. Reactive strategies are pattern based, anomaly based or hybrid, which can be applied at host/victim network or intermediate network, i.e., routers and switch. Anomalybased methods are mostly used for detection of unknown attacks [4]. Statistical methods use network statistics as determining factor of anomaly detection. These methods are fast, easily deployable and suitable for handling huge amount of data; hence, solutions for DDoS detection on Cloud are generally based on statistical anomaly.

Leu and $\mathrm{Li}$ [5] proposed a cumulative sum algorithm based DDoS prevention system. It detects sudden increment in network traffic and cooperatively detects attacks by delivering detection statistics to other filters. It has a high detection rate. Hop count filtering (HCF) by Wang et al [6] is a filtering scheme that investigates the hop count of incoming packet to check whether it is legitimate or not. After detecting DDoS attack, it discards spoofed packets. It is easy to deploy but prone to distributed unspoofed attacks. PacketScore [7] is a packet filtering scheme based on statistical anomaly detection against DDoS attacks. It distinguishes the attack traffic from legitimate traffic by utilizing the concept of conditional legitimate probability. Confidence-based filtering (CBF) method by Chen et al [8] is a DDoS detection method, which enhances PacketScore in terms of handling large amount of traffic. Legitimate packets are collected from attack-free traffic to create a nominal profile. In the attack period, CBF score for each packet is calculated and compared to the nominal profile. If conditions are satisfied the packet is accepted, otherwise discarded. Determining the weights of different attribute pairs used for profile is a key issue in this method.

Vieira et al [9] proposed the Grid and Cloud Computing Intrusion Detection System. It uses a designed audit system for detection of attacks that are undetected by network and host-based systems. It integrates rule-based and anomalybased analysis to detect specific intrusions. An event auditor in each node can identify local attacks and alerts other nodes. Lo et al [10] proposed a method that uses cooperative IDS for exchanging alert messages. IDS, cooperatively using majority vote, decides whether the alert message is trustworthy or not. Failure of single point is averted by this design of the IDS system.

VMFence [11] provided a management framework to introduce flexibility for Cloud service provider. The Cloud provider can configure detection rules for each domain according to the type of service running in each VM. Dynamic adjustment of number of detection modules is done with the number of VMs. Oktay and Sahingoz [12] used the gateway as a proxy for network intrusion detection job. All networks in traffic pass through the gateway to remove additional bottlenecks because of IDS's network traffic. The detector is set up on a separate VM located on the gateway. The Cloud trace back model by Joshi et al [13], which is based on deterministic packet marking algorithm, is able to filter attack traffic as well as trace back to the real source of attack.

The proposed work in this paper is mostly motivated from the research in DDoS cited by Mirkovic and Reiher [3], cumulative sum algorithm [5] and Oktay and Sahingoz's network intrusion detection job on gateway [12].

In this work, a DDoS detection framework is proposed and designed for Cloud, which has high detection rate and few false positives. This model is able to detect unspoofed as well as spoofed distributed attacks, is free from single point of failure and is scalable. Main objectives of this work are the following:

- to design an algorithm to identify DDoS attacks with high accuracy in real time.

- to design the framework for DDoS detection in Cloud and

- to evaluate the proposed model in Cloud and with datasets.

\section{Proposed framework and algorithm for DDOS attack detection}

This paper proposes a statistical anomaly detection mechanism and filtering model based on distributed networks for fast and real-time DDoS attack detection in Cloud. Due to multi-tenancy and self-provisioning properties of Cloud, traditional DDoS detection techniques to detect attack cannot be applied. Multi-tenancy in Cloud can lead to insider attack in the network. When multiple tenants are running their services in the Cloud, attacker can pose as a tenant and can use the same resources to create DDoS attack from the internal network itself. Traditional techniques focus more on outsider attacks in case of DDoS. When load on the servers increases, in the normal scenario, self-provisioning ensures smooth functioning by automatically provisioning new nodes for the service. However, due to DDoS attacks, network load is highly increased, which causes an infinite recursive increment in self-provisioning in its attempt to improve the utilization and subsequently wasting the resources. With unlimited provisioning, it causes huge financial implications as well. Traditional techniques consider networks to have upper bound on resources and insider attacks to be less probable and easily controllable.

In the proposed method, value distributions of various network attribute pairs are used to make it more complex to mimic. We observe from experiments that these value distributions do not follow any specific pattern, but attribute pairs of the same host or network show similar kind of 
behaviour with respect to time. We make a distributed model of this scheme in the Cloud, because different distributions for different hosts make it very hard for the intruder to mimic the behaviour of a host using the data form compromised host.

\subsection{Proposed framework of filter in Cloud}

The framework of DDoS filter in Cloud is shown in figure 1. In Cloud, Multiple DDoS filters are distributed in different hosts or tenants. On each host there are two types of nodes, one coordinator node and other normal VMs. Each VM generates a weekly local profile and shares this profile with the coordinator node. This coordinator node generates an averaged profile for new or migrated VMs.

State diagram of these nodes is shown in figure 2. Initially all VMs are in uninitialized state. On instantiation of
VMs, a new state is reached. When these new nodes generate or receive profiles, they change their state to existing. A single node is selected from existing nodes and its state is changed to coordinator. This selection can be static or a dynamic election algorithm [14] can be used. VMs change their state to migrated when migration from one host to other happens, which can reach existing state after receiving the profile.

Main components of DDoS filter are a profile generator, packet filter, average profile generator and node monitor. The interaction diagram of these components is shown in figure 3 .

3.1a Profile generator: This component of DDoS filter is deployed on normal nodes. It generates profile for all individual nodes by collecting normal user behaviour and shares the profile with coordinator node at regular intervals. Profile generation is explained in detail in section 3.2. It

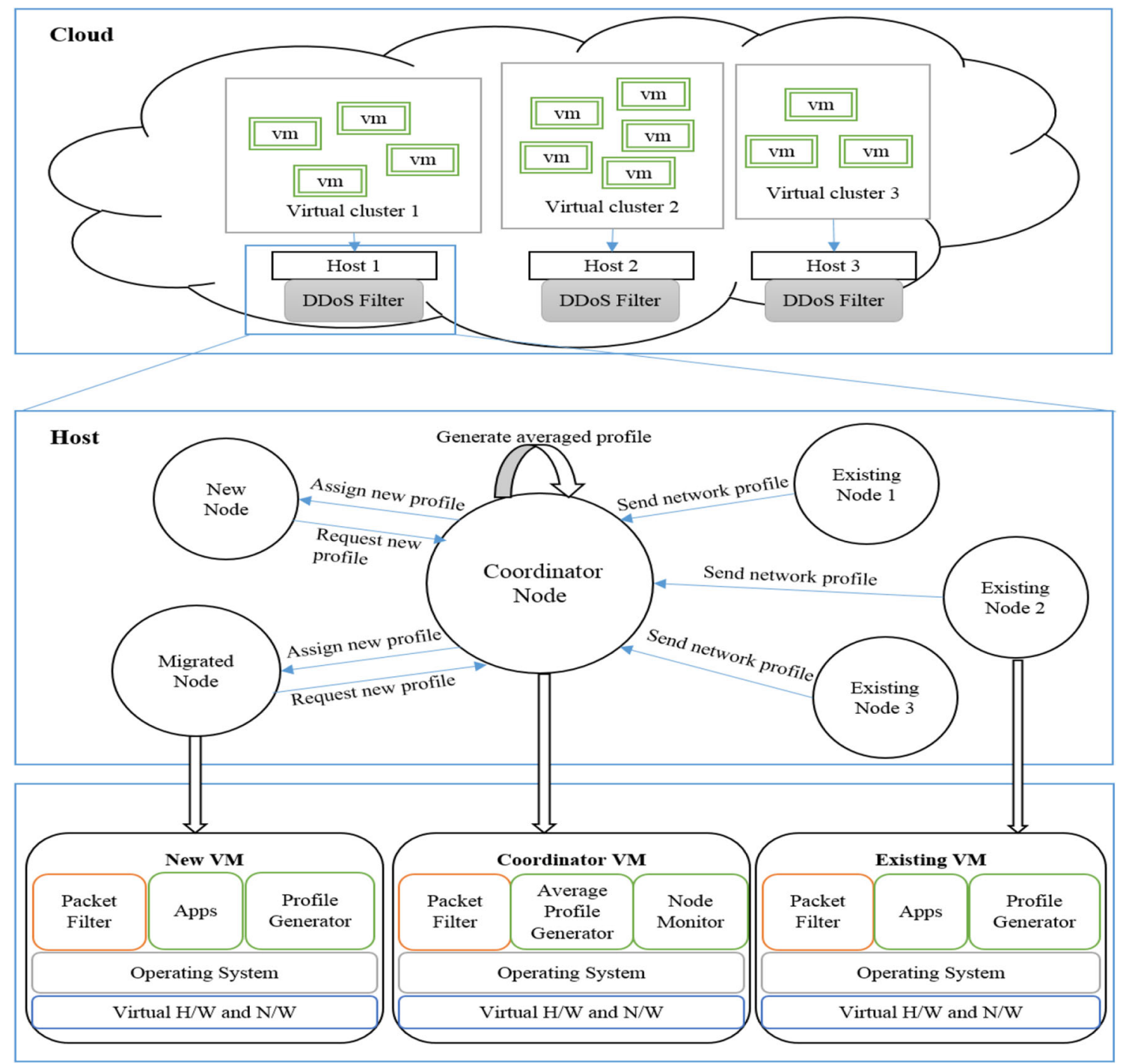

Figure 1. Framework for DDoS filter in Cloud environment. 


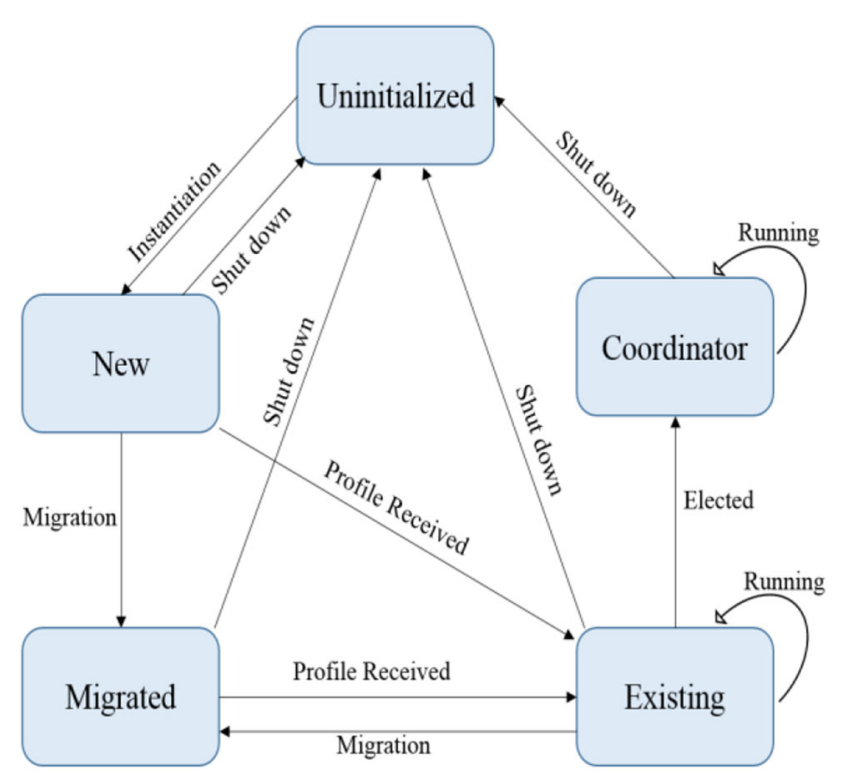

Figure 2. State diagram of virtual machines.

also shares the updated profile by packet filter with coordinator node. In new and migrated nodes it sends a request for new averaged profile to coordinator node. If the coordinator fails, it detects the failure and sends an alert to administrator to instantiate a new coordinator node. Since it is a distributed algorithm that works on individual nodes for collection of complete network traffic, we require profile generator at each node of the service to be protected.

3.1b Packet filter: This is the main module that filters the incoming traffic at each node of the network. This component of DDoS filter is also deployed on all the nodes. First it checks the availability of the profile and waits till the profile generator creates a new profile or obtains it from the coordinator. It monitors incoming packets in all individual nodes and generates alerts or logs. If a deviation from normal local profile is detected, packets are marked as attack packet. In the proposed algorithm, deviation is calculated from a dependency ranking. For non-attack packets it updates the profile. Packet filtering is explained in detail in section 3.2.

3.1c Average profile generator: It is located in coordinator nodes and it generates an averaged profile for individual host from the profiles shared by VMs at regular intervals. It also generates the normal profile for coordinator nodes. The averaged profile is assigned to new VMs, so that these nodes can detect attacks during intrusive periods without involving the profile generation phase. In individual nodes, profile of one week is used for detection, which is updated weekly or updated with daily replacement with corresponding day of previous week in weekly profile.

3.1d Node monitor: This is required for coordination on the distributed modules at each step, i.e., adding or removing a new node, checking status of the average profile generator and checking health of the modules in the nodes. This component of DDoS filter is located in the coordinator node. It registers the VMs, when a profile is shared or requested by them. It monitors the activity of packet filters in registered VMs and alerts the administrator on the failure of any filter.

\subsection{Proposed packet filtering algorithm}

The proposed packet filtering algorithm for individual node is shown in figure 4. This method uses the supervised learning technique to detect abnormal behaviour and it

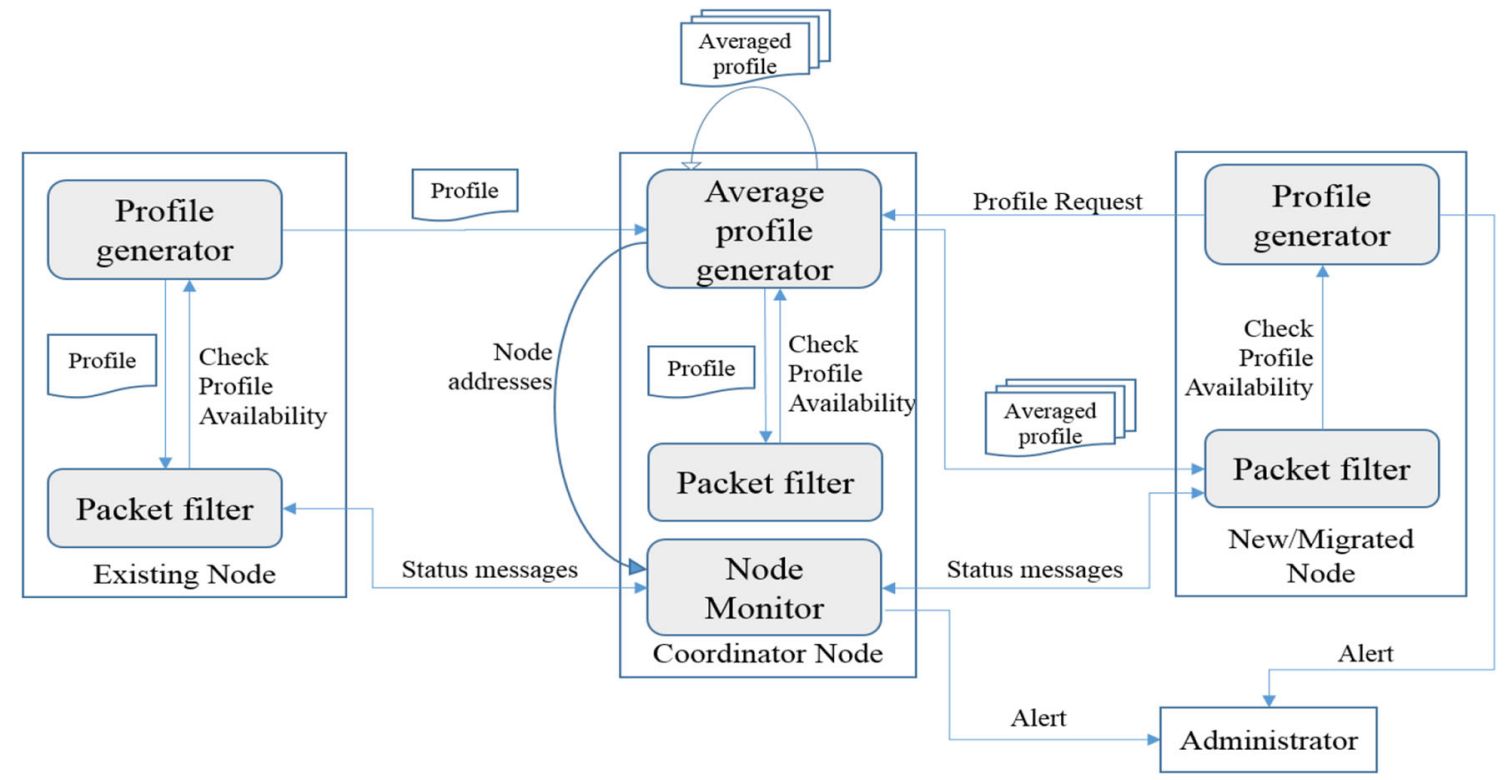

Figure 3. Interaction diagram of DDoS filter components. 


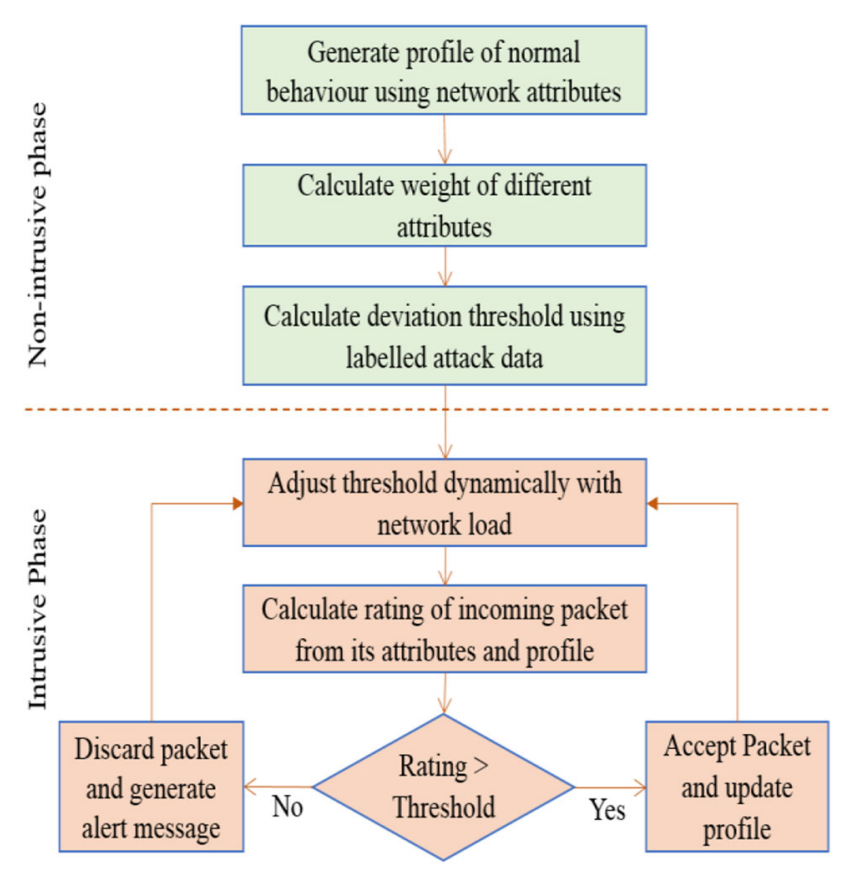

Figure 4. Packet filtering algorithm.

works in two phases: non-intrusive phase and intrusive phase. The profile generator and packet filter components of DDoS filter apply to non-intrusive phase and intrusive phase of this algorithm, respectively.

3.2a Non-intrusive phase: In the non-intrusive phase or profile generation phase, the DDoS detector is trained in a period of no attack. In this phase, attack detector learns the normal traffic pattern of network. This traffic pattern is a function of multiple network attributes. We have selected six most representative attributes of network for our purpose. Individually, these attributes are not sufficient to determine traffic patterns; some pairs of these attributes are useful to determine logical traffic patterns. A collective profile of normal behaviour is created using selected attribute pairs. Network profiles can be created by shifting window of daily, weekly or monthly traffic. Kim et al [7] suggest that weekly profile is the most suitable to determine user or network behaviour due to sufficient data points as well as optimized data load. Different attribute pairs have different amounts of impact on the profile; hence weight of attribute pairs is also calculated from the mutual information and confidence between pairs. Then a threshold is calculated from data with labelled attack to measure the deviation from normal behaviour. Weights of attribute pairs and thresholds are dynamic in nature, which ensures identifying ever-changing network behaviour.

3.2b Intrusive phase: In intrusive phase and updating phase, packet filters work in distributed network with a possibility of attack. Each incoming packet on each host is rated based on attribute weights and their values in dynamic weekly profile. Each incoming packet is marked as attack packet or nonattack packet by measuring the deviation from normal profile. Higher rating of a packet shows that it is more legitimate. Lower rating of a packet means it might be an attack packet. Marked packets are sent as feedback to the profile generator, which ensures that only normal traffic is used in determination of attribute pairs' weight. For non-attack packets the collective profile is updated. The deviation threshold can be static or dynamically adjusted with network load [15].

\section{Experimental results}

In this section, experiments are discussed that support the effectiveness and the accuracy of proposed approach. For the performance analysis of proposed DDoS attack detector, the DARPA intrusion detection evaluation dataset DARPA 1999 [16] is used and various DDoS attacks in Cloud environment using OpenNebula [17] are also simulated.

\subsection{Experimental set-up for testing in Cloud environment}

4.1a Set-up: A private Cloud was set up for this experiment using the OpenNebula Cloud management tool for simulation of DDoS attacks in real Cloud environment. A test scenario is shown in figure 5 . A web server is set up on host 1 and a FTP server on host 2 of Cloud. Multiple instances of VMs are used for both the servers in non-intrusive period. VMs VM12, VM15 and VM16 are used for web server and VM11, VM17 and VM18 are used for initial profile creation. VM11 and VM12 are coordinator nodes for FTP server and web server, respectively. Additional VMs VM19 and VM20 are instantiated in intrusive period to simulate new VMs' detection against attacks.

4.1b Method: In this experiment, six attributes from transport and network layer headers are selected for attack detection. These features are TCP flags, time to live value, source IP address, total length, source port and destination port number. We found these selected attributes as distinct values but they are not very useful for profiling. However, some of the pairs of these attributes provide good insight of the network. For example, the source IP with TCP flags is useful to predict a non-human behaviour if requests of high frequency from the same source also terminate quickly. How much these attribute pairs are useful is determined by the weight assigned to these during profile generation phase. Based on these six features, fifteen different attribute pairs are created. To generate the profile, confidence values of attribute pairs are stored as described in Chen et al [8]. Mutual information is used for the ranking of individual features or attributes. Mutual information measures 


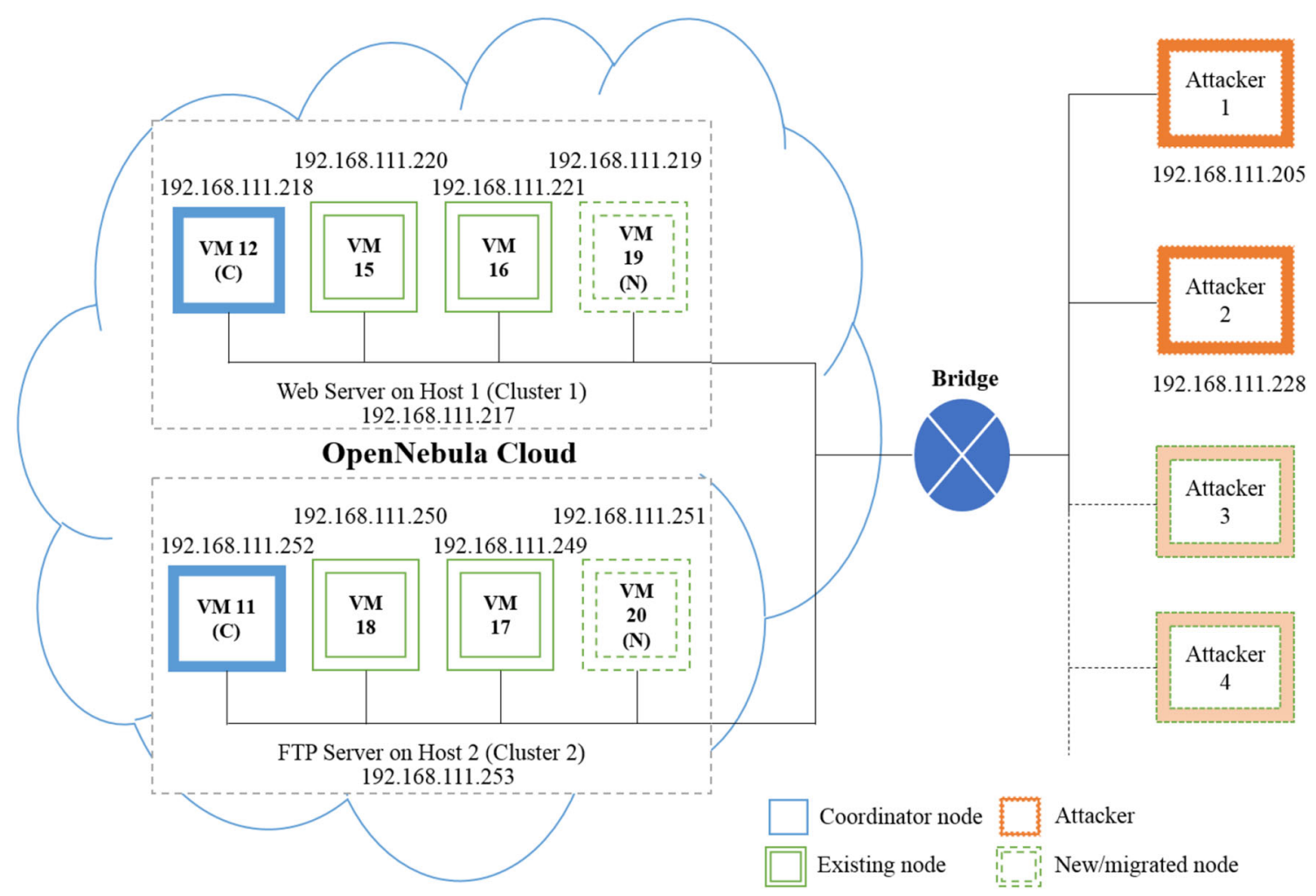

Figure 5. Testing scenario for DDoS attack detection in OpenNebula Cloud. A web server is created on host 1 and a FTP server is created on host 2. Multiple attacker machines outside the Cloud are used for attack on these servers.

dependence between variables or attributes [18] and identifies the importance of an attribute pair occurring together [19]. Mutual information between $\mathrm{Ai}$ and $\mathrm{Aj}$ from their respective value vectors $\mathrm{Vi}$ and $\mathrm{Vj}$ in the given sample is calculated as follows:

$$
\mathrm{MI}(\mathrm{Ai}, \mathrm{Aj})=\sum_{\mathrm{A} i \in V i} \sum_{\mathrm{Aj} \in V j} C(\mathrm{Ai}, \mathrm{Aj}) \log \frac{C(\mathrm{Ai}, \mathrm{Aj})}{C(\mathrm{Ai}) C(\mathrm{Aj})} .
$$

Here, $\mathrm{Ai}$ is the $i$ th value of attribute $\mathrm{A}$ and $C(\mathrm{Ai})$ is its confidence value from sample $\mathrm{S}$. Later, a dependency rating of incoming packets, which is weighted average of confidence of attribute pairs, is calculated as follows:

$$
\text { rating }=\frac{\sum_{\mathrm{Ai}, \mathrm{Aj} \in \mathrm{S}} \mathrm{MI}(\mathrm{Ai}, \mathrm{Aj}) C(\mathrm{Ai}, \mathrm{Aj})}{\sum_{\mathrm{Ai}, \mathrm{Aj} \in \mathrm{S}} M I(\mathrm{Ai}, \mathrm{Aj})} .
$$

This rating is always in the range $[0,1]$. Higher rating of a packet shows that it is more legitimate. Lower rating of a packet means it might be an attack packet.

4.1c Attack model: DDoS attack from multiple Linux and Windows machines were simulated. Different types of DDoS attacks as shown in table 1 were carried out from outside as well as from one host to another host in Cloud using a Low Orbit Ion Cannon tool (LOIC) [20]. These attacks were carried out for a $4.5 \mathrm{~h}$ time period in random time intervals.
Table 1. Different attack types defined.

\begin{tabular}{lccc}
\hline Type & Protocol & Victim host & $\begin{array}{c}\text { Insider } \\
\text { attack? }\end{array}$ \\
\hline Type 1 & TCP & Web server & No \\
Type 2 & TCP & FTP server & No \\
Type 3 & HTTP & Web server & No \\
Type 4 & TCP & Web server & Yes \\
Type 5 & TCP & Double-intensity attack on FTP & No \\
& & server & \\
\hline
\end{tabular}

\subsection{Accuracy and scalability of filters in Cloud environment}

Performance of DDoS attack filter in different VMs against various attacks is shown in table 2 . By observing the results it is analysed that very low false positive rates and acceptable false negative rates are achieved with the proposed model. Newly created VMs VM19 and VM20 are also able to detect attacks with acceptable rates. DDoS attacks from both inside and outside were detected. True positive rate and accuracy based on previous data are shown in table 3.

Multiple false positive and true positives values are displayed in table 4 against random values of rating parameter from Eq. (2). Based on these parameters we plot 
Table 2. Performance of DDoS detectors against various attacks in Cloud.

\begin{tabular}{lccccc}
\hline VM ID & Total packets & Total attack packets & False positive $(\%)$ & False negative $(\%)$ & Attack types \\
\hline VM12 & 17668 & 0 & 0.25 & NA & None \\
VM15 & 226213 & 58815 & 1.71 & 3.67 & Type 1,3 \\
VM16 & 487045 & 136372 & 1.99 & 4.15 & Type 1,3 \\
VM19 & 216354 & 30289 & 1.16 & NA & Type 4 \\
VM11 & 15904 & 0 & 0.29 & 3.16 & None \\
VM18 & 198023 & 29703 & 1.76 & 6.56 & Type 2 \\
VM17 & 574284 & 166542 & 0.83 & NA & Type 2, 5 \\
VM20 & 197277 & 0 & & None \\
\hline
\end{tabular}

Table 3. True positive rate and accuracy against DDoS attack in Cloud.

\begin{tabular}{lcccccc}
\hline VM ID & False positive & True positive & False negative & True negative & True positive rate $(\%)$ & Accuracy $(\%)$ \\
\hline VM15 & 2863 & 56656 & 2159 & 164535 & 96.33 & 97.78 \\
VM16 & 6978 & 129349 & 7023 & 343695 & 94.85 & 97.13 \\
VM19 & 2158 & 28999 & 1290 & 183907 & 95.74 & 96.84 \\
VM18 & 2962 & 28764 & 939 & 165358 & 93.44 & 98.03 \\
VM17 & 7502 & 155617 & 10925 & 400240 & NA & 96.79 \\
VM20 & 1637 & 0 & 0 & 195640 & NA & 99.17 \\
VM11 & 46 & 0 & 0 & 15858 & NA & 99.71 \\
VM12 & 44 & 0 & 0 & 17624 & 99.75 \\
\hline
\end{tabular}

Table 4. True positive and false positives against rating parameter.

\begin{tabular}{ccccc}
\hline $\begin{array}{l}\text { Value (rating } \\
\text { parameter) }\end{array}$ & $\begin{array}{c}\text { Attack } \\
\text { packets }\end{array}$ & $\begin{array}{c}\text { Non-attack } \\
\text { packets }\end{array}$ & $\begin{array}{c}\text { False } \\
\text { positives }\end{array}$ & $\begin{array}{c}\text { True } \\
\text { positives }\end{array}$ \\
\hline 0.009 & 421721 & 1511047 & 24192 & 399385 \\
0.001 & 421721 & 1511047 & 29678 & 399749 \\
0.010 & 421721 & 1511047 & 344961 & 400847 \\
0.100 & 421721 & 1511047 & 455880 & 401210 \\
0.400 & 421721 & 1511047 & 717503 & 407284 \\
0.700 & 421721 & 1511047 & 1300762 & 414293 \\
0.000 & 421721 & 1511047 & 0 & 0 \\
1.000 & 421721 & 1511047 & 1511047 & 421721 \\
\hline
\end{tabular}

the receiver operating characteristic curve (AUC) as shown in figure 6.

When the load is increased on any server, it instantiates new VMs for that server. With instantiation or migration of VM from one host to another, VM successfully obtains new profiles from the coordinator and scalability requirement of DDoS detector is satisfied.

\subsection{Performance analysis using DARPA dataset}

4.3a Dataset: DARPA intrusion detection evaluation dataset 1999 is used for testing against simple denial of service attacks. The DARPA dataset is a collection of packet trace data from an airbase research laboratory. This dataset

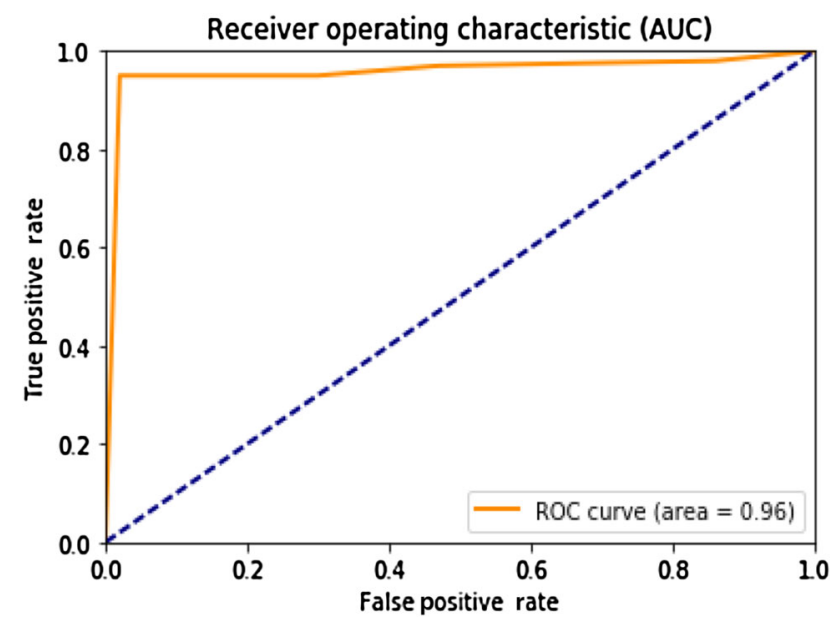

Figure 6. ROC AUC curve for DDOS detection model.

contains 3 weeks of training data and 2 weeks of testing data. Weeks 1 and 3 contain data with no attacks and week 2 data contain data with labelled attacks. Testing data contain various instances of attacks. These attacks are divided in four categories: denial of service, user to root, remote to local attacks and probes. The proposed scheme is evaluated against denial of service attacks, which include attacks like syn flood, smurf, tcpreset, ping flood, etc.

4.3b Method: First, all packet traces in the dataset are preprocessed and frequency values of 6 attributes and attribute pairs mentioned in section $4.1 \mathrm{~b}$ are extracted. Later the 

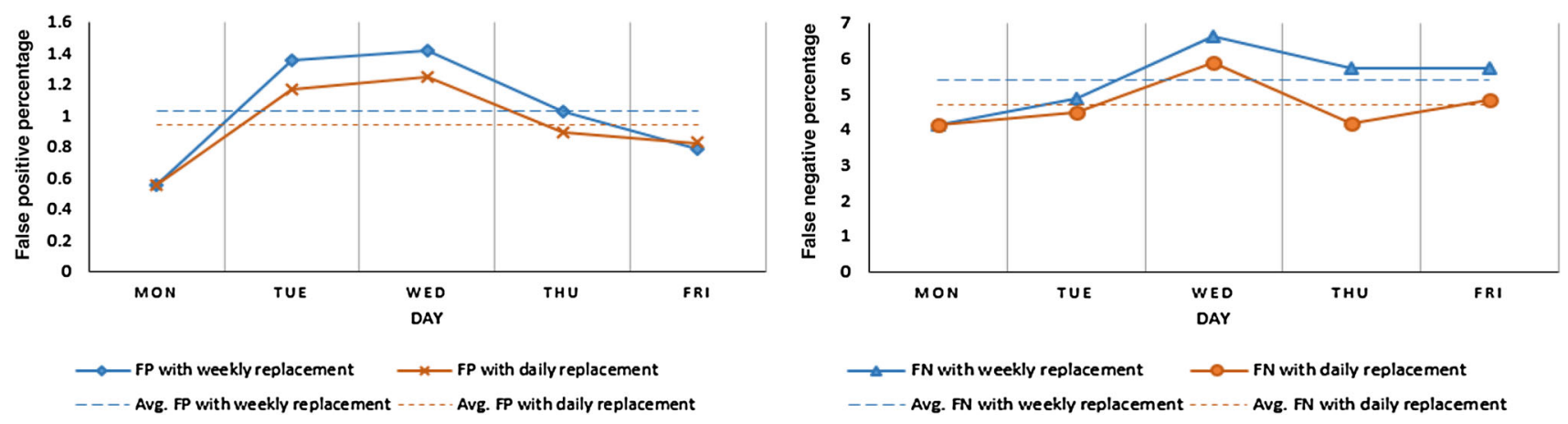

Figure 7. Comparison of false positive rate and false negative rate of DDoS detector using weekly profile with weekly replacement vs. daily replacement.

proposed algorithm is applied on these data. Network profile is created from week 1 data and threshold is calculated from week 2 data. This threshold is calculated such that it can detect the maximum number of attacks with false positive rate less than $1 \%$. From this experiment, the threshold value is obtained as 0.009 . Then the profile is updated from week 3 data ignoring attack packets. Testing is done on week 4 data using this profile. The performance of algorithm is evaluated using weekly profile with both weekly replacement and daily replacement.

\subsection{Accuracy and efficiency with DARPA dataset}

The performance of DDoS attack filter against different attacks is shown in figure 7. It can be observed from the graphs that profile with daily replacement is better than profile with weekly replacement for higher detection rates. It can also be observed that false positive rates are very low and false negative rates are in acceptable range for the proposed DDoS detection algorithm.

In this experiment, average size of generated profiles is $627 \mathrm{kB}$ and 1-6 MB of physical memory is used in runtime; these storage requirements are very low as compared with memory availability of present systems. These statistics support the memory and storage efficiency of this algorithm.

\section{Conclusion}

This paper proposed a statistical and distributed packet filtering algorithm and framework for detection of DDoS attacks in Cloud. This scheme is able to handle DDoS attacks with high accuracy and high detection rate. While statistical approach of this model provides high speed of detection, distributed nature of filtering scheme improves scalability and flexibility. Robustness is ensured by using a coordinator node, which constantly monitors other nodes. Performance of the proposed method is evaluated using the dataset and in Cloud. This packet filtering model is able to detect attacks from outside as well as from one tenant to another in the Cloud. All these features and its real-time detection ability make this scheme suitable for defence against DDoS attacks in Cloud environment.

\section{References}

[1] Ries B 2010 Hackers' most destructive attacks, http://www. thedailybeast.com/articles/2010/ 12/11/hackers-10-most-famous-attacks-worms-and-ddos-takedowns.html

[2] Clark J 2011 Amazon cloud used in PlayStation Network hack. http://www.zdnet.com/amazon-cloud-used-in-playsta tion-network-hack-4010022454/

[3] Mirkovic J and Reiher P 2004 A taxonomy of DDoS attack and DDoS defense mechanisms. ACM SIGCOMM Comput. Commun. Rev. 34(2): 39-53

[4] Modi C, Patel D, Borisaniya B, Patel H, Patel A and Rajarajan M 2013 A survey of intrusion detection techniques in cloud. J. Netw. Comput. Appl. 36(1): 42-57

[5] Leu F Y and Li Z Y 2009 Detecting DoS and DDoS attacks by using an intrusion detection and remote prevention system. In: Proceedings of IAS, pp. 251-254

[6] Wang H, Jin C and Shin K G 2007 Defense against spoofed IP traffic using hop-count filtering. IEEE/ACM Trans. Netw. 15(1): 40-53

[7] Kim Y, Lau W C, Chuah M C and Chao H J 2006 PacketScore: a statistics-based packet filtering scheme against distributed denial-of-service attacks. IEEE Trans. Depend. Secure Comput. 3(2): 141-155

[8] Chen Q, Lin W, Dou W and Yu S 2011 CBF: a packet filtering method for DDoS attack defense in cloud environment. In: Proceedings of the 9th IEEE International Conference on Dependable, Autonomic and Secure Computing (DASC), pp. 427-434

[9] Vieira K, Schulter A, Westphall C and Westphall C M 2010 Intrusion detection for grid and cloud computing. IT Profess. 12(4): $38-43$

[10] Lo C C, Huang C C and Ku J 2010 A cooperative intrusion detection system framework for cloud computing networks. In: Proceedings of the 39th International Conference on Parallel Processing Workshops (ICPPW), pp. 280-284 
[11] Jin H, Xiang G, Zou D, Wu S, Zhao F, Li M and Zheng W 2013 A VMM-based intrusion prevention system in cloud computing environment. J. Supercomput. 66(3): 1133-1151

[12] Oktay U and Sahingoz O K 2013 Proxy network intrusion detection system for cloud computing. In: Proceedings of the International Conference on Technological Advances in Electrical, Electronics and Computer Engineering (TAEECE), pp. 98-104

[13] Joshi B, Vijayan A S and Joshi B K 2012 Securing cloud computing environment against DDoS attacks. In: Proceedings of the International Conference on Computer Communication and Informatics (ICCCI), pp. 1-5

[14] Ingram R, Shields P, Walter J E and Welch J L 2009 An asynchronous leader election algorithm for dynamic networks. In: Proceedings of the IEEE International Symposium on Parallel and Distributed Processing (IPDPS), pp. 1-12
[15] Kasera S, Pinheiro J, Loader C, Karaul M, Hari A and LaPorta T 2001 Fast and robust signaling overload control. In: Proceedings of the 9th International Conference on Network Protocols, pp. 323-331

[16] DARPA 1999 Intrusion Detection Evaluation Data Set, http://www.1l.mit.edu

[17] Milojičić D, Llorente I M and Montero R S 2011 OpenNebula: a cloud management tool. IEEE Internet Comput. 15(2)

[18] Guyon I and Elisseeff A 2003 An introduction to variable and feature selection. J. Mach. Learn. Res. 3: 1157-1182

[19] Torkkola K 2003 Feature extraction by non-parametric mutual information maximization. J. Mach. Learn. Res. 3: $1415-1438$

[20] LOIC 2012 Low orbit ion cannon, http://sourceforge.net/ projects/loic 